\title{
A Simple Approach for Enhancing the Output Performance of Solar-Pumped Solid-State Lasers
}

\author{
Dawei Liang and Rui Pereira \\ Centro de Física e Investigação Tecnológica (CEFITEC), Departamento de Física, Faculdade de Ciências e Tecnologia (FCT), \\ Universidade Nova de Lisboa, Campus de Caparica, 2829-516 Caparica, Portugal \\ Correspondence should be addressed to Dawei Liang, dl@fct.unl.pt
}

Received 19 September 2008; Accepted 2 December 2008

Recommended by David Coutts

A simple truncated fused silica elliptical cavity is proposed to enhance the output performance of solar-pumped solid-state lasers. The imaging property of the truncated elliptical cavity ensures an enhanced absorption distribution within an Nd:YAG rod. Optimum pumping parameters are found through ZEMAX nonsequential ray-tracing and LASCAD laser cavity analyses. Compared with the output laser performance of a 3D-compound parabolic concentrator-2D-compound parabolic concentrator (3D-CPC-2D-CPC) cavity, the truncated cavity provides $11 \%$ more multimode and $72.7 \%$ more $\mathrm{TEM}_{00}$ laser powers. A laser beam of high beam quality can be produced efficiently. The standard tracking error for multimode laser power is also reduced to only $4.0 \%$ by the truncated cavity.

Copyright $\odot 2009$ D. Liang and R. Pereira. This is an open access article distributed under the Creative Commons Attribution License, which permits unrestricted use, distribution, and reproduction in any medium, provided the original work is properly cited.

\section{Introduction}

Solar-pumped lasers convert broadband solar radiation into coherent and narrowband laser emissions. Since the appearance of the first solar-pumped laser [1], researchers have been attempting to improve the output performance such as collection efficiency and beam quality [2-6]. Tracking errorinduced laser power decrease is also a major concern [7], since heliostat tracking error shifts the center of absorption inside the laser rod and the resonator stability depends on how well the sun is tracked. The technologies of photovoltaics and diode-pumped solid-state lasers have separately attracted much attention and achieved industrial maturity. The direct pumping of a solid-state laser from sunlight to laser light saves two energy conversion steps and is potentially more efficient. However, much less consideration has been devoted to direct solar-pumped lasers and the technology is currently much less mature. Solar-pumped laser is, however, much simpler and more reliable due to the complete elimination of electrical power generation and power conditioning equipment. Space-based applications for solar lasers hold the most promise. $6.7 \mathrm{~W} / \mathrm{m}^{2}$ collection efficiency has been achieved by pumping the Nd:YAG rod within the 3Dcompound parabolic concentrator-2D-compound parabolic concentrator (3D-CPC-2D-CPC) cavity [6]. The recent progress in high-efficiency and economical solar-pumped laser with a Fresnel lens and a chromium codoped ceramic laser rod has largely improved the collection efficiency to $18.7 \mathrm{~W} / \mathrm{m}^{2}$ [8]. Researchers, including us, are now strongly motivated to build a powerful solar-pumped laser for the efficient reduction of magnesium $(\mathrm{Mg})$ from magnesium oxide $(\mathrm{MgO})$. Large amounts of heat and hydrogen $\left(\mathrm{H}_{2}\right)$ are given off from the reaction of magnesium with water. $\mathrm{Mg}$ can be an alternative to fossil fuel. But for a magnesium combustion engine to function as a practical source of energy, the lasers need to be powered by a renewable energy source, such as solar power.

The imaging property of an elliptical cavity has already been widely explored in solid-state lasers, contributing to the improvement of laser beam quality [9-11]. An input face of the truncated cavity is firstly positioned to the focal area of a primary parabolic mirror. By optical refraction at the air/fused silica interface, a concentrated focal spot with large rim angle is then converted into a divergent spot with 
small rim angle within the fused silica material, facilitating its further concentration into the laser rod by the truncated cavity. ZEMAX nonsequential ray-tracing and LASCAD laser cavity analyses confirm both the laser efficiency and the beam quality advantages of the truncated cavity. Large standard tracking error for multimode power from the 3D-CPC2D-CPC cavity is considerably reduced by the truncated cavity.

\section{Numerical Analysis of the 3D-CPC-2D-CPC Solar Laser System}

The astigmatic corrected target aligned (ACTA) solar concentrator system [6] provided an efficient pumping approach, enabling the convenient placement of the laser system on a horizontal optical table. The segmented primary mirror of $3.4 \mathrm{~m}$ diameter and $17 \mathrm{~m}$ radius of curvature was mounted on a two-axis heliostat programmed to track the sun continuously and project the reflected sunlight toward the entrance plane of a secondary concentrator via a plane folding mirror. The double-stage secondary concentrator consisted of a first-stage 3D-CPC followed by a second-stage 2D-CPC, as shown in Figure 1(a). Concentrated solar light at $11.5^{\circ}$ half-angle cone entered the $100 \mathrm{~mm}$ diameter entrance aperture of the 3D-CPC, which in turn funneled the light out of its $24 \mathrm{~mm}$ diameter exit aperture at $55^{\circ}$ half-angle cone. The emitted light entered the $33 \times 24 \mathrm{~mm}^{2}$ aperture of the 2D-CPC, as illustrated in Figure 1(b), illuminating an antireflection end-coated 1.1\% Nd:YAG laser rod, $6 \mathrm{~mm}$ in diameter and $72 \mathrm{~mm}$ in length. The laser rod was mounted inside a quartz flow tube along the 2D-CPC axis. The laser resonator design was plane-parallel. The rear mirrors were coated with a high reflectivity dielectric coating of $\mathrm{R}>99.7 \%$. Output mirrors with different reflectivity, varying between $80 \%$ and $98 \%$, were tested individually. For the first mirror with $6.85 \mathrm{~m}^{2}$ collection area, $45 \mathrm{~W}$ laser power was achieved by using a $95 \%$ reflectivity output mirror.

ZEMAX nonsequential ray tracing and LASCAD laser cavity software was used to evaluate numerically the laser performance of the 3D-CPC-2D-CPC solar laser system. The dimensions of the first-stage ACTA solar collector were utilized in the nonsequential ray tracing software. The profiles of both the 3D-CPC and the 2D-CPC were essential for designing both the axially symmetric 3D-CPC concentrator and the 2D-CPC pumping cavity. The 3DCPC-2D-CPC cavity designed in AUTO-CAD software was then exported to ZEMAX software. The laser rod, the cooling water, and the flow tube were also dimensioned. Several aspects are important for the correct numerical analysis of the 3D-CPC-2D-CPC solar laser system.

The Solar Spectra, the Tabulated Model of Absorption for Nd: YAG Material, and the Overlap between the Nd:YAG Absorption Spectra and the Solar Spectra. The standard solar spectra [12] for one and a half air mass (AM1.5) were used as the reference data for consulting the spectral irradiance $\left(\mathrm{W} / \mathrm{m}^{2} / \mathrm{nm}\right)$ at each wavelength. The irradiance cumulative integral of the whole solar spectra equals to the typical terrestrial value of $900 \mathrm{~W} / \mathrm{m}^{2}$, which agrees well with the experimental data in [6].

For 1.1\% Nd:YAG laser medium, 22 absorption peaks were defined in ZEMAX software. The central wavelengths of these peaks are $527 \mathrm{~nm}, 531 \mathrm{~nm}, 568 \mathrm{~nm}, 578 \mathrm{~nm}, 586 \mathrm{~nm}$, $592 \mathrm{~nm}, 732 \mathrm{~nm}, 736 \mathrm{~nm}, 743 \mathrm{~nm}, 746 \mathrm{~nm}, 753 \mathrm{~nm}, 758 \mathrm{~nm}$, $790 \mathrm{~nm}, 793 \mathrm{~nm}, 803 \mathrm{~nm}, 805 \mathrm{~nm}, 808 \mathrm{~nm}, 811 \mathrm{~nm}, 815 \mathrm{~nm}$, $820 \mathrm{~nm}, 865 \mathrm{~nm}$, and $880 \mathrm{~nm}$. The highest absorption coefficient reaches $\alpha=10 \mathrm{~cm}^{-1}$, while the lowest is about $\alpha=1.5 \mathrm{~cm}^{-1}$. The averaged FWHM absorption bandwidth of each peak is about $1 \mathrm{~nm}$ [13]. All the above peak wavelengths and their respective absorption coefficients were added to the glass catalog for Nd:YAG material in ZEMAX software.

Solar irradiance values corresponding to the abovementioned 22 peak absorption wavelengths for the Nd:YAG medium, ranging from $527 \mathrm{~nm}$ to $880 \mathrm{~nm}$, could be consulted from the standard solar spectra for AM1.5 and saved as source wavelength data in ZEMAX software. In order to reduce the thermal load on the laser rod in the ACTA solar laser system, the IR radiation which does not contribute to laser pumping is filtered out by the $45^{\circ}$ filter residing between the folding mirror and the entrance plane of the 3D-CPC-2D-CPC concentrator. The same filter eliminates also the radiation below $500 \mathrm{~nm}$ to prevent the creation of color centers within the rod. Only the source wavelength data within $527 \mathrm{~nm}-880 \mathrm{~nm}$ range are, therefore, analyzed in ZEMAX software. The undesirable UV+Visble (less than $500 \mathrm{~nm}$ ) and IR (more than $880 \mathrm{~nm}$ ) radiation were not included in the ray-tracing analysis. For the terrestrial insolation value of $900 \mathrm{~W} / \mathrm{m}^{2}$, $6165 \mathrm{~W}$ solar power reaches the ACTA mirror with $6.85 \mathrm{~m}^{2}$ collection area. If $14 \%$ overlap between the Nd:YAG absorption spectra and the solar spectra is considered [3], $864 \mathrm{~W}$ total absorbable solar power is then attributed to a $3.4 \mathrm{~m}$ diameter circular light source, representing absorbable incoming radiation to the ACTA mirror, in ZEMAX software. $0.27^{\circ}$ solar half-angle is assumed in the ray-tracing analysis.

Optical Parameters for the Components of the ACTA Solar Laser System. To numerically analyze the ACTA solar laser system, $85 \%$ reflectivity for the first-stage ACTA mirror, 90\% reflectivity for the folding mirror, and 95\% reflectivity for all the other reflector surfaces of the 3D-CPC-2D-CPC cavity were used in ZEMAX analysis. The quartz flow tube has $1 \mathrm{~mm}$ wall thickness. Three is a $1.5 \mathrm{~mm}$ gap between the flow tube and the rod for cooling water. The side surface of both the rod and the flow tube is modeled as uncoated. The solar spectra absorption coefficients for both water and the quartz flow tube are also defined accordingly. The $6.0 \mathrm{~mm}$ diameter cylindrical rod is divided into a total of 40000 zones. During ray tracing, the path length in each intercepted zone is found. With this value and the absorption coefficients of the 22 peak absorption wavelengths, the total power absorbed by the rod can be calculated by summing up the absorbed pump radiations of all the zones within the rod. The absorbed powers for the rods of other diameters are also analyzed. 


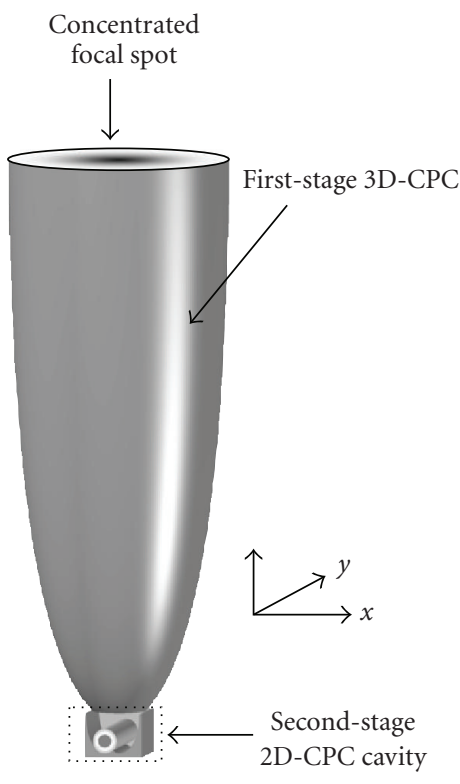

(a)

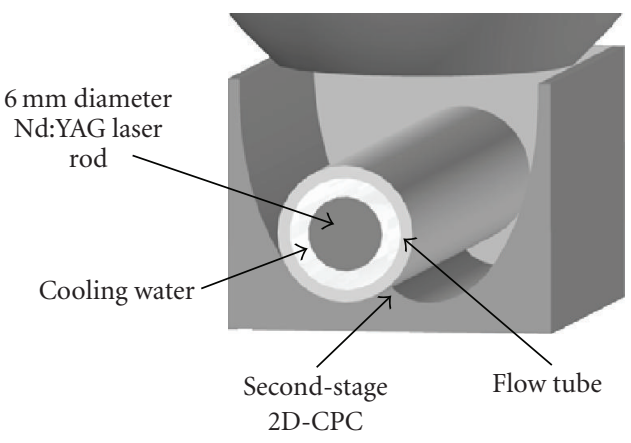

(b)

Figure 1: (a) Double-stage 3D-compound parabolic concentrator-2D-compound parabolic concentrator (3D-CPC/2D-CPC) pumping cavity. (b) Enlarged view of the 2D-CPC cavity with the laser rod of $6 \mathrm{~mm}$ diameter and $50 \mathrm{~mm}$ length.

Ray propagation within the rod is three dimensional. However, the number of rays propagating along the rod axis direction is assumed equal on average. The ray distribution along the rod axis is uniform as a result. As a typical illustration, we choose the central cross-sectional absorption distribution perpendicular to the rod axis. The gray-scale central cross-sectional absorption distribution of the $6.0 \mathrm{~mm}$ diameter rod, pumped by the 3D-CPC-2D-CPC cavity, is given in Figure 2. Black signifies near maximum absorption, whereas white signifies little or no absorption. The 3DCPC-2D-CPC cavity does not provide a near-Gaussian absorption profile. There exists low absorption density in the central core region. The ray-tracing analysis indicates that there is also a strong lack of rotational symmetry and the maximum occurs on the sides of the rod. The absorption distribution pumped by the 3D-CPC-2D-CPC cavity is also represented by the absorbed flux/volume distributions along both the central cross-section row and the central cross-section column of the rod, as shown in Figure 2, indicating also a lack of absorbed flux in the central rod region.

The angular tracking error of a commercial heliostat may reach $2 \mathrm{mrad}$ [14]. For $8.5 \mathrm{~m}$ focal distance, $2 \mathrm{mrad}$ error corresponds to $17 \mathrm{~mm}$ transversal shift from the ideal focal position of the ACTA mirror. If the focal spot moves in the direction parallel to the laser rod axis, the strong pump absorption distribution will be shifted correspondingly along the rod in the opposite axial direction, causing only minor reduction in output laser power. If the focal spot moves in the direction perpendicular to the rod axis (the transversal shift as we named), the strong absorption distribution within the rod will be shifted laterally in the direction perpendicular to the rod axis, causing significant reduction

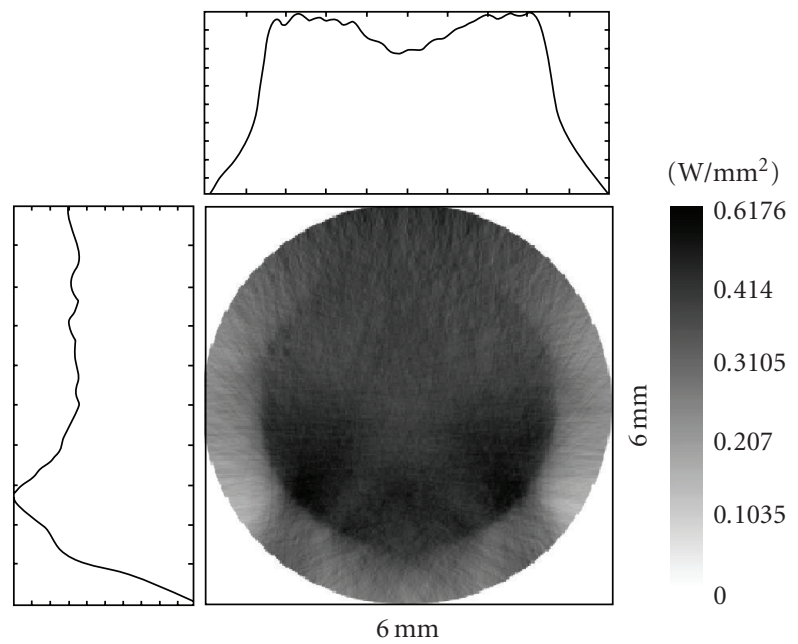

FIGURE 2: Gray-scale absorption distribution for the $6 \mathrm{~mm}$ diameter Nd:YAG rod pumped by the 3D-CPC-2D-CPC cavity. Black signifies near or maximum absorption whereas white signifies little or no absorption (vertical unit in $\mathrm{W} / \mathrm{mm}^{2}$ ).

in laser output power. It is, therefore, sensible to concentrate only on the transversal shift of the focal spot in raytracing analysis. Pumped by the concentrated light spot with $17 \mathrm{~mm}$ transversal shift along the input plane, the gray-scale absorption distribution for the $6.0 \mathrm{~mm}$ diameter rod is illustrated in Figure 3. Significant difference is found between the absorption distributions without and with tracking errors, as illustrated in Figures 2 and 3, respectively. The transversal shift to the right side causes the dislocation of absorbed pump power to the left bottom side of the rod. 


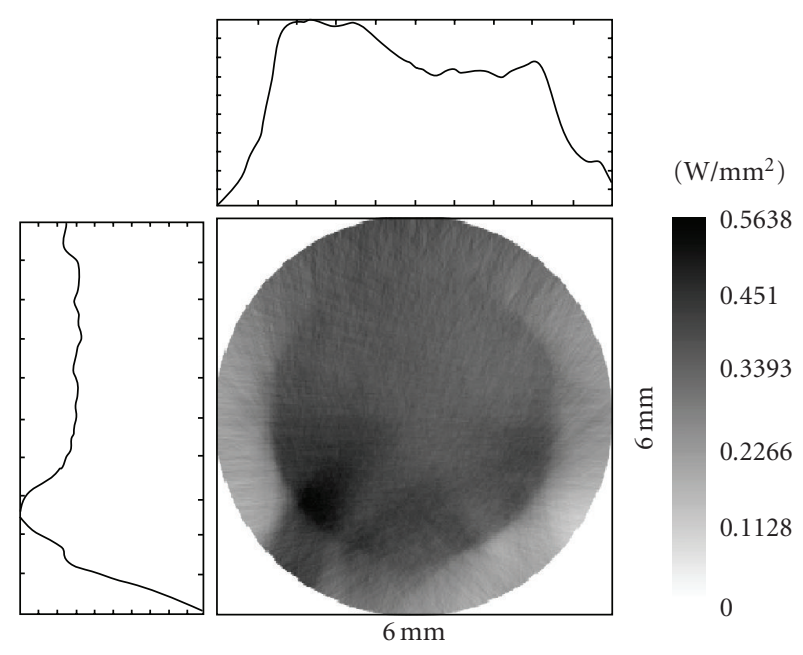

FIGURE 3: Gray-scale absorption distribution for the $6 \mathrm{~mm}$ diameter $\mathrm{Nd}$ :YAG rod within the 3D-CPC-2D-CPC cavity (angular tracking error $2 \mathrm{mrad}$, vertical unit in $\mathrm{W} / \mathrm{mm}^{2}$ ).

Besides, the absorption distribution is neither rotationally nor axially symmetric.

ZEMAX ray-tracing code is used to maximize the absorbed pump flux within the rod. The absorbed pump flux data from the ZEMAX analysis is then processed by LASCAD software. Output couplers of different reflectivity, ranging from $80 \%$ to $98 \%$, are tested individually to maximize the multimode laser power. A plane-parallel laser resonator of $450 \mathrm{~mm}$ length, an averaged solar pump wavelength of $660 \mathrm{~nm}$, and a round-trip loss of 5.0\% [3] are used in the LASCAD analysis. For the $6 \mathrm{~mm}$ diameter and $72 \mathrm{~mm}$ length $\mathrm{Nd}$ :YAG rod, $47 \mathrm{~W}$ laser power is achieved by adopting a $95 \%$ reflectivity output coupler, matching well with the published experimental data of $45 \mathrm{~W}$ [6]. This result validates also our estimate on the overlap between the solar emission spectra and the Nd:YAG absorption spectra.

In LASCAD simulation, $45 \mathrm{~W}$ output laser power is optimized to $57.3 \mathrm{~W}$ by using the $6 \mathrm{~mm}$ diameter laser rod of only $50 \mathrm{~mm}$, rather than $72 \mathrm{~mm}$ length. The round-trip loss is consequently reduced from $5.0 \%$ to $3.6 \%$. There is still enough space for both mounting and cooling the $\mathrm{Nd}$ :YAG rod of $50 \mathrm{~mm}$ length through the 2D-CPC cavity of $33 \mathrm{~mm}$ length. To evaluate the laser performance of the 3D-CPC-2D-CPC cavity, the Nd:YAG rods of 3, 4, 5, and $6 \mathrm{~mm}$ diameters are tested separately within the 3D-CPC2D-CPC cavity. Output coupler of different reflectivity is also tested separately to optimize the output laser power from each rod. The absorbed pump powers, the multimode, and the $\mathrm{TEM}_{00}$ laser powers are listed in Table 1. We define the standard tracking error as the power decrease resulting from a 2 mrad tracking error divided by the laser power without tracking error. For the $17 \mathrm{~mm}$ shift along the input plane of the 3D-CPC-2D-CPC cavity, the standard tracking errors are given in italics in Table 1 . With the increase of rod diameter, there exist gradual increases in both absorbed pump power and multimode laser power. The $\mathrm{TEM}_{00}$ laser power is, however, adversely affected. A small diameter rod
TABLE 1: Laser output performance and the respective standard $2 \mathrm{mrad}$ tracking errors for the laser rods of different diameters, pumped by the 3D-CPC-2D-CPC cavity.

\begin{tabular}{lcccc}
\hline Diameter $(\mathrm{mm})$ & $3 \mathrm{~mm}$ & $4 \mathrm{~mm}$ & $5 \mathrm{~mm}$ & $6 \mathrm{~mm}$ \\
\hline $\mathrm{P}_{\mathrm{ABS} .}(\mathrm{W})$ & 129.5 & 182.0 & 229.2 & 264.6 \\
Standard tracking error (\%) & 6.7 & 6.5 & 6.4 & 6.3 \\
$\mathrm{P}_{\mathrm{M}-\mathrm{M}}(\mathrm{W})$ & 36.6 & 47.9 & 55.2 & 57.3 \\
Standard tracking error (\%) & 8.7 & 8.9 & 9.3 & 9.9 \\
$\mathrm{P}_{\mathrm{TEM}}(\mathrm{W})$ & 5.5 & 4.0 & 2.9 & 2.2 \\
Standard tracking error (\%) & 8.6 & 8.7 & 9.0 & 9.2 \\
\hline
\end{tabular}

is effective in producing $\mathrm{TEM}_{00}$ laser power. Relatively stable and large standard tracking errors are dominant phenomena in the 3D-CPC-2D-CPC cavity. As rod diameter increases, there exists a slow increase in standard tracking errors for output laser powers. The reasons for these variations will be discussed in Section 4.

The 3D-CPC-2D-CPC cavity is commonly designed according to the edge-ray principle of nonimaging optics [15]. The CPC is used to convert the radiation from a large area homogenous source emitting uniformly into small angles, to a small area homogenous output emitting uniformly into large angles, thus the étendue is preserved. This preservation implies that irradiance is larger at the output surface than at the input surface, leading to a net concentration of the pump radiation. The 3D-CPC-2D-CPC solar laser pumping system has, therefore, the advantage of the efficient collection and concentration of pump power from the large area input surface of the $3 \mathrm{D}$-CPC concentrator to the small area rod surface of the Nd:YAG medium. As seen from Figures 2 and 3, edge-ray design methods are, however, too constrained to provide the desired absorption distribution within the central core region of the laser rod, leading to reduced laser beam quality. There are nevertheless numerous applications of when the strong concentration of pump energy within the central laser rod volume is beneficial in laser technology. It is, therefore, desirable to search for other cavity designs that can produce efficient laser operation and laser beam of high beam quality.

\section{Truncated Fused Silica Elliptical Cavity}

A heliostat with a plane mirror and a parabolic mirror are generally combined to pump a solar laser. In direct tracking mode, the parabolic mirror is mounted directly onto the heliostat. A laser head is mounted at the focal area of the parabolic mirror. Direct tracking features high collection efficiency. A small plane folding mirror can be applied to invert the incoming solar radiation so that the laser head can be installed a little more conveniently. In indirect tracking mode, a large plane mirror is mounted on a heliostat which redirects the incoming solar radiation to a stationary parabolic mirror. The laser head can be conveniently mounted at the focal area of the parabolic mirror. Easy laser resonator alignment is ensured. Both the heliostat and the parabolic mirrors can be easily installed on 
the same height [3]. Due to the large plane mirror required for redirecting the incoming rays from different incident angles, low collection efficiency is revealed by indirect tracking mode. The 3D-CPC-2D-CPC approach provides, on the other hand, advanced and high-cost technology for achieving the high collection efficiency. The simple and low-cost heliostat plane mirror and parabolic mirror combination is nevertheless still preferred in solar-pumped laser research. Due to the compactness of the truncated cavity and its consequent easy fitting in the focal area of the parabolic mirror, direct tracking mode is analyzed here to ensure high collection efficiency. Our analysis is also valid for the simple heliostat plane mirror and stationary parabolic mirror pumping approach.

The elliptical cavity is based on the geometrical theorem that rays originating from one focal line of an elliptical cylinder are reflected to the other focal line. As a consequence of the preservation of angles, the portion of the elliptical reflector nearer the rod forms a reduced image of the pump source [16]. For the truncated elliptical cavity mounted in the focal area of the parabolic mirror, as shown in Figure $4(\mathrm{a})$, the concentrated rays of $60^{\circ}$ rim angle are firstly refracted into the divergent rays of $36^{\circ}$ rim angle within the fused silica cavity. As a result of the significant reduction in incident rim angle, from $60^{\circ}$ to $36^{\circ}$, only the portion of the elliptical reflector near the crystal and the two end faces are finally used to focus tightly the pump radiations along the central core region of the laser rod.

Some practical considerations are important for the ray-tracing analysis. $900 \mathrm{~W} / \mathrm{m}^{2}$ solar irradiance is used as ZEMAX analysis. By using the 3.4 diameter and $1.32 \mathrm{~m}$ focal distance parabolic mirror, mounted directly on the two-axis heliostat in direct tracking mode, $6020 \mathrm{~W}$ solar power is concentrated into a $13.6 \mathrm{~mm}$ FWHM diameter focal spot on the entrance plane of the truncated cavity. The focal spot has a near-Gaussian profile with $19.8 \mathrm{~W} / \mathrm{mm}^{2}$ maximum power density, resulting in a temperature high enough for melting metal. It is, however, still far below the $1665^{\circ} \mathrm{C}$ softening point for fused silica material. Even without active cooling, fused silica light guide and fibres have already been successfully used to transport high flux solar radiation without any damage to the optical material $[17,18]$. The utility of fused silica material for the proposed approach is hence ensured. High optical purity (99.9999\%) fused silica material can be used to manufacture the truncated cavity, as shown in Figure 4(b). The truncated input face is coplanar with the first focal line and perpendicular to the major axis of the truncated elliptical cavity. A $9 \mathrm{~mm}$ diameter cylindrical cooling channel is opened along the second focal line. Both the input face and the inner surface of the cooling channel of the cavity are optically polished to allow for the highest transparency. The highly concentrated focal spot can easily damage antireflection coatings and all the polished surfaces are therefore assumed as uncoated in ZEMAX analysis. The curved elliptical cavity surface and the two parallel end faces are also optically polished and gold coating is finally applied (not shown in Figure 4(b)). A specularly reflective fused silica pump cavity is hence formed. The pump wavelengths above $500 \mathrm{~nm}$ are efficiently reflected to the rod, while the wavelengths below $500 \mathrm{~nm}$ are poorly reflected and absorption occurs at the cavity walls. A small dielectric or metallic multilayer plane folding mirror [19], for example, a cold mirror, is mounted near the focal area of the parabolic mirror. Useful solar radiation (from $500 \mathrm{~nm}$ to $880 \mathrm{~nm}$ ) is reflected to the truncated cavity while infrared heat (larger than $880 \mathrm{~nm}$ ) is transmitted away from the folding mirror. The efficient cooling to the rod, the truncated cavity, and the plane folding mirror are of vital importance. The laser rod is mounted between two crystal holders, supported by two water-cooled end blocks, between which the truncated cavity is mounted.

The maximum absorbed pump radiation and the most favorable absorption distribution within the rod are two crucial factors for optimizing the truncated cavity. The matching between the absorption spectra of $1.1 \%$ Nd:YAG medium and the solar radiation spectra is analyzed, as mentioned in the previous section. Identical to the 3D-CPC-2D-CPC system, $85 \%$ folding mirror angle-averaged reflectivity, $90 \%$ parabolic mirror reflectivity, 95\% cavity reflectivity are also utilized. The central cross-sectional absorption distribution perpendicular to the rod axis is also examined. The crosssectional gray-scale absorption distribution for the $6 \mathrm{~mm}$ diameter Nd:YAG rod, pumped by the truncated cavity, is given in Figure 5. Black signifies near maximum absorption, whereas white signifies little or no absorption. The truncated cavity does provide a near-Gaussian absorption profile. The ray-tracing analysis indicates that there is high degree of rotational symmetry and the maximum absorption occurs along the central core region of the rod. The cross-sectional absorption profiles pumped by the truncated cavity are represented by the absorbed flux/volume distribution along both the central cross-section row and the central crosssection column of the $6 \mathrm{~mm}$ diameter rod, as shown in Figure 5, indicating the strong concentration of absorbed flux in the central core region. The optimized truncated elliptical cavity has finally the following dimensions: $a=$ $25 \mathrm{~mm}, b=19 \mathrm{~mm}$, and $h=32 \mathrm{~mm}$.

The $2 \mathrm{mrad}$ angular tracking error is also used for the two-axis heliostat upon which a primary parabolic concentrator of $3.4 \mathrm{~m}$ diameter and $1.32 \mathrm{~m}$ focal distance are mounted, resulting in $2.656 \mathrm{~mm}$ transversal shift from the ideal focal position. Similar to the previous section, we concentrate here only on the standard tracking error analysis for the transversal shift of the focal spot. Figure 6 shows the gray-scale cross-sectional absorption distribution for the $6.0 \mathrm{~mm}$ diameter Nd:YAG rod. The symmetric absorption distribution in Figure 5 is slightly shifted away from the central core region.

The optimized absorbed pump flux data from the raytracing analysis is then processed by LASCAD software for optimizing the multimode laser power in a plane-parallel laser resonator of $450 \mathrm{~mm}$ length and 3.6\% round-trip loss. The averaged solar pump wavelength of $660 \mathrm{~nm}$ is also utilized. Output coupler reflectivity is used for maximizing the multimode laser power. Nd:YAG rods of different diameters are analyzed individually. The absorbed pump powers, the multimode, and the TEM 00 laser powers are listed in Table 2. The standard tracking error for each laser power is listed 


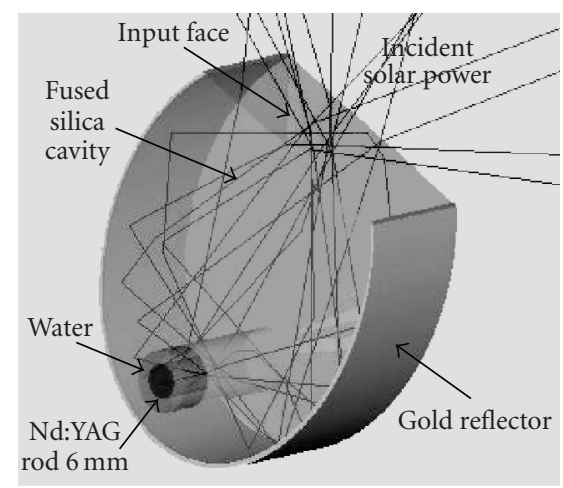

(a)

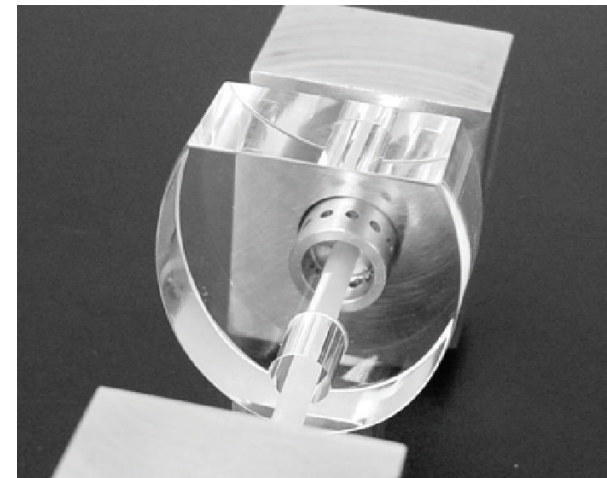

(b)

Figure 4: (a) Three-dimensional view of the truncated cavity (only 10 trace rays are illustrated in the figure). (b) Apreliminary experimental setup of the truncated cavity is shown. Both the curved elliptical surface and the side faces are still uncoated.

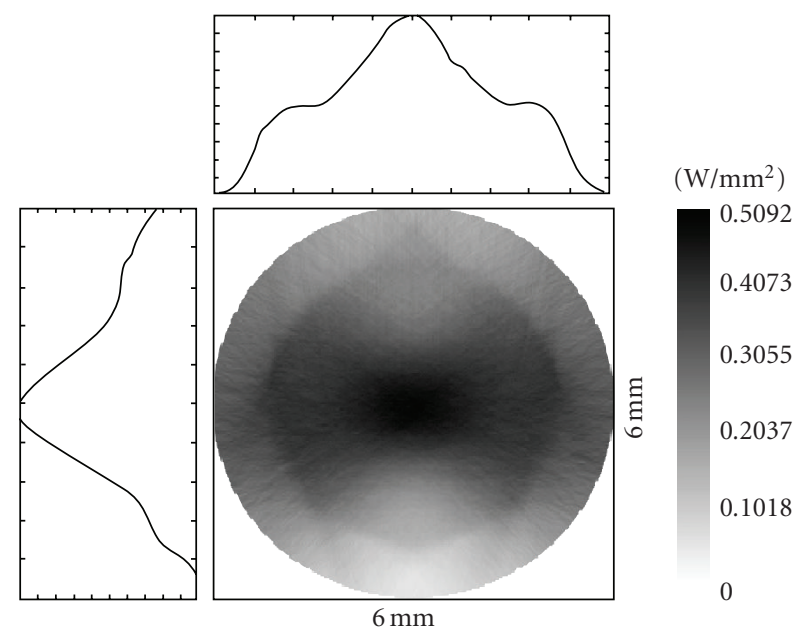

FIGURE 5: Gray-scale absorption distribution for the $6 \mathrm{~mm}$ diameter $\mathrm{Nd}$ :YAG rod within the truncated cavity (Vertical unit in $\mathrm{W} / \mathrm{mm}^{2}$ ).

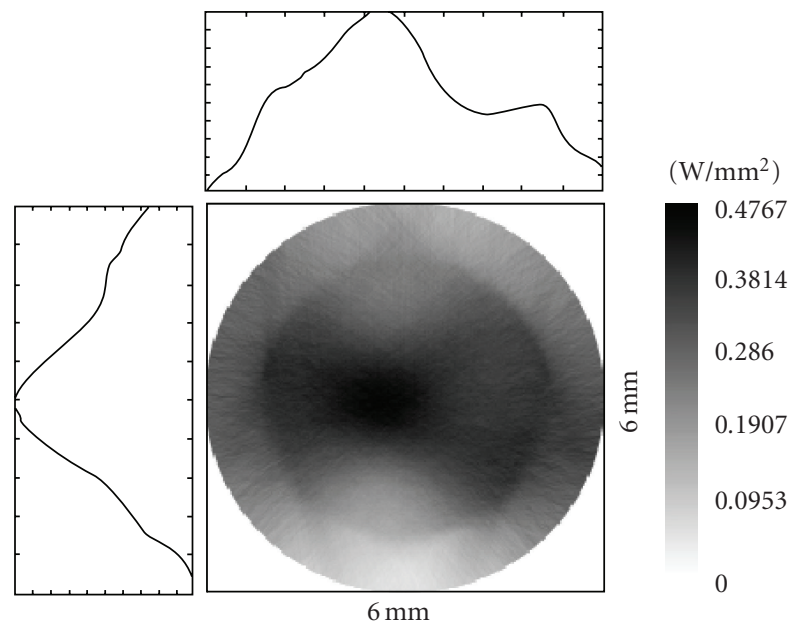

FIGURE 6: Gray-scale absorption distribution for the $6 \mathrm{~mm}$ diameter Nd:YAG rod within the truncated cavity (angular tracking error $2 \mathrm{mrad}$, vertical unit in $\mathrm{W} / \mathrm{mm}^{2}$ ).
TABLE 2: Laser output performances and the respective standard 2 mrad tracking errors for the laser rods of different diameters, pumped by the truncated cavity.

\begin{tabular}{lcccc}
\hline Diameter $(\mathrm{mm})$ & $3 \mathrm{~mm}$ & $4 \mathrm{~mm}$ & $5 \mathrm{~mm}$ & $6 \mathrm{~mm}$ \\
\hline $\mathrm{P}_{\mathrm{ABS} .}(\mathrm{W})$ & 136.2 & 185.9 & 230.2 & 269.7 \\
Standard tracking error (\%) & 5.5 & 4.0 & 3.2 & 2.7 \\
$\mathrm{P}_{\mathrm{M}-\mathrm{M}}(\mathrm{W})$ & 41.0 & 52.0 & 59.1 & 63.6 \\
Standard tracking error (\%) & 7.1 & 5.4 & 4.4 & 4.0 \\
$\mathrm{P}_{\mathrm{TEM}_{00}}(\mathrm{~W})$ & 7.1 & 5.7 & 4.7 & 3.8 \\
Standard tracking error (\%) & 11.5 & 11.1 & 9.7 & 9.5 \\
\hline
\end{tabular}

below each respective power value. With small diameter rod, high-order modes are suppressed by large diffraction losses and beam quality improves. High pumping density or small rod surface is attractive but challenging for the 3D-CPC2D-CPC approach. The total absorbed pump powers within small diameter rods are, however, largely improved by the truncated cavity, as compared by both Tables 1 and 2 .

\section{Comparisons between the 3D-CPC-2D-CPC Cavity and the Truncated Fused Silica Elliptical Cavity}

4.1. 3D-CPC-2D-CPC Cavity. The ray-tracing analysis indicates that the first-stage $3 \mathrm{D}-\mathrm{CPC}$ causes the major transmission loss. Besides, the $17 \mathrm{~mm}$ transverse shift of focal spot along the entrance plane of the first 3D-CPC concentrator results in $6 \%$ extra transmission loss. Independent of the rod diameter used for capturing the pump radiation within the secondary 2D-CPC cavity, the 6\% extra pump power has already been lost in the first-stage. This explains why the standard tracking error for the 3D-CPC-2D-CPC cavity remains relatively large and stable. Large diameter rod traps slightly more pump power, so there is a slow reduction in the standard tracking error, varying between $6.7 \%$ and $6.3 \%$, as the rod diameter increases from $3 \mathrm{~mm}$ to $6 \mathrm{~mm}$. The transverse shift of the focal spot increases the output angles of the rays exiting from the $3 \mathrm{D}-\mathrm{CPC}$ concentrator. 
The incident angles of the rays entering the 2D-CPC cavity are also increased. By observing the edge-ray principle in nonimaging optics [15], the absorbed pump power within the rod is shifted further away from the central core region, as shown in Figure 3. The concentration of the absorbed pump power in the peripheral zones of the rod is increased. Only a large diameter rod is capable of absorbing all the tracking error-dependent pump radiations. The laser power from the large diameter rod is hence sensitive to the tracking errors. Small diameter laser rod captures less pump power, missing especially the tracking error-dependent pump radiation located away from the rod axis. Small diameter rod is therefore less sensitive to tracking errors. This explains why the tracking error for the laser powers in Table lincreases slowly with the increase of rod diameters.

4.2. Truncated Fused Silica Elliptical Cavity. Compared with the multimode performances of the 3D-CPC-2D-CPC cavity, as listed in Table 1, there exists a general improvement in multimode powers for the rod of all diameters when the truncated cavity is applied, as listed in Table 2. With the increase of rod diameter, the standard tracking error is reduced from $7.1 \%$ to $4.0 \%$. Due to the imaging properties of the elliptical cavity, the concentrated focal spot without or with tracking errors can all be concentrated exactly or approximately in the central core region of the $6 \mathrm{~mm}$ diameter rod, as shown in Figures 5 and 6 . The absorbed pump power and the output laser power for large diameter rod are less sensitive to the transversal movement of the focal spot. Any transversal shift of the spot can, however, cause significant variation of absorbed pump power for small diameter rod. This explains why the standard tracking error for the multimode laser powers is gradually reduced with the increase of rod diameter.

Figure 1 and Table 1 indicate that the 3D-CPC-2D-CPC cavity is not effective in providing the absorbed pump power within the central core region, producing significantly less $\mathrm{TEM}_{00}$ power. Due to the large enhancement in the absorbed power within the central core region, as shown in Figure 5, the strong increase in $\mathrm{TEM}_{00}$ laser power is easily achieved by the truncated cavity. The imaging property of the elliptical cavity signifies that any shift of the focal spot along the input face causes the inevitable variation of the absorbed pump power within the central core region. The standard tracking error for $\mathrm{TEM}_{00}$ laser power from the truncated cavity is hence large, varying from $11.5 \%$ to $9.5 \%$, as shown in Table 2. The standard tracking error from the 3D-CPC$2 \mathrm{D}-\mathrm{CPC}$ cavity is also relatively large, varying from $8.6 \%$ to 9.2\%, as shown in Table 1 .

The truncated cavity constitutes an effective alternative to the 3D-CPC-2D-CPC cavity. The laser output performance such as laser power and beam quality can be significantly enhanced by this simple pumping approach. The standard tracking error for the truncated cavity compares also favorably with the 3D-CPC-2D-CPC cavity. Table 3 summarizes the advantages of the truncated cavity. The positive values in bold represent the enhancements in the absorbed pump powers, in the multimode and, significantly, in the TEM $\mathrm{T}_{00}$
TABLE 3: Enhancements in the absorbed pump powers, the laser output powers, and the standard tracking errors achieved by the truncated cavity. Comparisons are made with the 3D-CPC-2D-CPC cavity. The positive values in bold signify the enhancement in the powers in percentage. The negative values in italic represent the reduction in standard 2 mrad tracking errors in percentage.

\begin{tabular}{lcccc}
\hline Diameter $(\mathrm{mm})$ & $3 \mathrm{~mm}$ & $4 \mathrm{~mm}$ & $5 \mathrm{~mm}$ & $\mathbf{6 m m}$ \\
\hline $\mathrm{P}_{\mathrm{ABS} .}$ enhancement $(\%)$ & $+\mathbf{5 . 2}$ & $\mathbf{+ 2 . 1}$ & $+\mathbf{0 . 4}$ & $+\mathbf{1 . 9}$ \\
Standard tracking error $(\%)$ & -1.2 & -2.5 & -3.2 & -3.6 \\
$\mathrm{P}_{\mathrm{M}-\mathrm{M}}$ enhancement $(\%)$ & $\mathbf{+ 1 2 . 0}$ & $\mathbf{+ 8 . 6}$ & $+\mathbf{7 . 1}$ & $+\mathbf{1 1 . 0}$ \\
Standard tracking error $(\%)$ & -1.6 & -3.5 & -4.9 & -5.9 \\
$\mathrm{P}_{\mathrm{TEM}} \mathrm{M}_{00}$ enhancement $(\%)$ & $\mathbf{+ 2 9 . 0}$ & $\mathbf{+ 4 2 . 5}$ & $+\mathbf{6 2 . 1}$ & $+\mathbf{7 2 . 7}$ \\
Standard tracking error $(\%)$ & +2.9 & +2.4 & +0.7 & +0.3 \\
\hline
\end{tabular}

laser powers. On the other hand, the negative values in italics represent the reduction in standard tracking errors. There exist a general enhancement in laser powers and an obvious reduction in standard tracking errors for multimode laser powers. The positive values in italics represent the deficiency of the truncated cavity, revealing slightly larger standard tracking errors for the $\mathrm{TEM}_{00}$ power than those by the $3 \mathrm{D}$ CPC-2D-CPC cavity.

The $M^{2}$ factors for the $6 \mathrm{~mm}$ diameter rod, pumped by the truncated cavity, are finally analyzed by LASCAD software. $M_{x}^{2}=49$ and $M_{y}^{2}=56$ are achieved for the $450 \mathrm{~mm}$ resonator length, representing a near-symmetric beam profile. The $M^{2}$ factors for the same rod, pumped by the 3D-CPC-2D-CPC cavity, are also analyzed. $M_{x}^{2}=$ 29 and $M_{y}^{2}=121$ are obtained for the same resonator length, revealing, however, a much more asymmetric beam profile. Laser beam brightness is the power divided by the product of beam spot area and its solid angle. That product is proportional to the product of $M_{x}^{2} M_{y}^{2}$ for asymmetric beam. Thus, we define a figure of merit $\mathrm{B}$, which is the laser power divided by $M_{x}^{2} M_{y}^{2}$. When the $6 \mathrm{~mm}$ diameter rod is pumped by the truncated cavity, $63.6 \mathrm{~W}$ laser power, $M_{x}^{2}=49$ and $M_{y}^{2}=56$ are obtained. The figure of merit B is $0.023 \mathrm{~W}$. When the same rod is pumped by the 3D-CPC-2D-CPC cavity, $57.3 \mathrm{~W}$ laser power, $M_{x}^{2}=29$ and $M_{y}^{2}=121$ are achieved. The figure of merit $\mathrm{B}$ is then reduced to $0.016 \mathrm{~W}$. The introduction of the truncated cavity favors, therefore, the efficient emission of high quality laser beam.

\section{Conclusions}

The truncated fused silica elliptical cavity is both simple and compact. ZEMAX nonsequential ray-tracing and LASCAD laser cavity analysis softwares are used to numerically calculate the laser output performance of the 3D-CPC-2D-CPC cavity, serving as an excellent reference for the numerical evaluation of the proposed truncated cavity. In terms of laser efficiency, Table 3 reveals a general enhancement in the absorbed pump powers and the laser powers. The best choice is obviously the $6 \mathrm{~mm}$ diameter rod. Compared with the optimized output performances of 3D-CPC-2D-CPC cavity, the truncated cavity offers $3.6 \%$ more absorbed pump power, 
$11 \%$ more multimode laser power and, significantly, $72.7 \%$ more $\mathrm{TEM}_{00}$ power. There exists also $5.9 \%$ reduction in the standard tracking error for multimode laser power. Besides, the truncated cavity improves largely the pump absorption distributions. There is considerably more absorbed pump power within the central core region and the absorption profile is more rotationally symmetric than the asymmetric absorption profile pumped by the 3D-CPC-2D-CPC cavity. The truncated elliptical pumping approach is hence effective for producing efficiently the laser beam of high beam quality.

\section{References}

[1] C. G. Young, "A sun-pumped cw one-watt laser," Applied Optics, vol. 5, no. 6, pp. 993-997, 1966.

[2] H. Arashi, Y. Oka, N. Sasahara, A. Kaimai, and M. Ishigame, "Solar-pumped cw $18 \mathrm{~W}$ Nd:YAG laser," Japanese Journal of Applied Physics, vol. 23, no. 8, pp. 1051-1053, 1984.

[3] M. Weksler and J. Shwartz, "Solar-pumped solid-state lasers," IEEE Journal of Quantum Electronics, vol. 24, no. 6, pp. 12221228, 1988.

[4] D. Cooke, "Sun-pumped lasers: revisiting an old problem with nonimaging optics," Applied Optics, vol. 31, no. 36, pp. 75417546, 1992.

[5] R. M. J. Benmair, J. Kagan, Y. Kalisky, et al., "Solar-pumped Er,Tm,Ho:YAG laser," Optics Letters, vol. 15, no. 1, pp. 36-38, 1990.

[6] M. Lando, J. Kagan, B. Linyekin, and V. Dobrusin, "A solar-pumped $\mathrm{Nd}$ :YAG laser in the high collection efficiency regime," Optics Communications, vol. 222, no. 1-6, pp. 371381, 2003.

[7] D. Liang, P. Bernardes, and R. Pereira, "Sun-pumped Nd:YAG laser with excellent tracking error compensation capacity," in Proceedings of the European Conference on Lasers and ElectroOptics (CLEO/Europe-EQEC '05), vol. 29B, Munich, Germany, June 2005, CA7-5-TUE.

[8] T. Yabe, T. Ohkubo, S. Uchida, et al., "High-efficiency and economical solar-energy-pumped laser with Fresnel lens and chromium codoped laser medium," Applied Physics Letters, vol. 90, no. 26, Article ID 261120, 3 pages, 2007.

[9] W. Koechner, Solid-State Laser Engineering, vol. 1 of Optical Sciences, chapter 6, Springer, Berlin, Germany, 4th edition, 1992.

[10] D. Liang and R. Pereira, "Diode pumping of a solid-state laser rod by a two-dimensional CPC-elliptical cavity with intervening optics," Optics Communications, vol. 275, no. 1, pp. 104-115, 2007.

[11] D. Liang, R. Pereira, and P. Bernardes, "Enhancing sunpumped laser performance by a truncated fused silica elliptical pump cavity," in Proceedings of the European Conference on Lasers and Electro-Optics and the International Quantum Electronics Conference (CLEO/Europe-IQEC'07), p. 1, Munich, Germany, June 2007, CA-7-MON.

[12] "ASTM G159-98 standard tables for references solar spectral irradiance at air mass 1.5: direct normal and hemispherical for a $37^{\circ}$ tilted surface," See for example: ASTM Standard G159, 1998.

[13] A. Brignon, G. Feugnet, J.-P. Huignard, and J.-P. Pocholle, "Compact Nd:YAG and Nd:YVO ${ }_{4}$ amplifiers end-pumped by a high-brightness stacked array," IEEE Journal of Quantum Electronics, vol. 34, no. 3, pp. 577-585, 1998.
[14] A. Kribus, I. Vishnevetsky, M. Meri, A. Yogev, and A. Sytnik, "Continuous tracking of heliostats," Journal of Solar Energy Engineering, vol. 126, no. 3, pp. 842-849, 2004.

[15] W. T. Welford and R. Winston, High Collection Nonimaging Optics, Academic Press, New York, NY, USA, 1989.

[16] S. B. Schuldt and R. I. Aagard, "An analysis of radiation transfer by means of elliptical cylinder reflectors," Applied Optics, vol. 2, no. 5, pp. 509-513, 1963.

[17] D. Liang, S. Duarte, J. Trindade, D. Ferreira, and L. F. Monteiro, "High-power solar energy transmission by solid-core fused silica light guides," in Nonimaging Optics: Maximum Efficiency Light Transfer VI, vol. 4446 of Proceedings of SPIE, pp. 76-87, San Diego, Calif, USA, August 2001.

[18] D. Liang, L. F. Monteiro, M. R. Teixeira, M. L. F. Monteiro, and M. Collares-Pereira, "Fiber-optic solar energy transmission and concentration," Solar Energy Materials and Solar Cells, vol. 54, no. 1-4, pp. 323-331, 1998.

[19] L. M. Fraas, J. E. Avery, and T. Nakamura, "Electricity from concentrated solar IR in solar lighting applications," in Proceedings of the 29th IEEE Photovoltaic Specialists Conference (PVSC '02), pp. 963-966, New Orleans, La, USA, May 2002. 

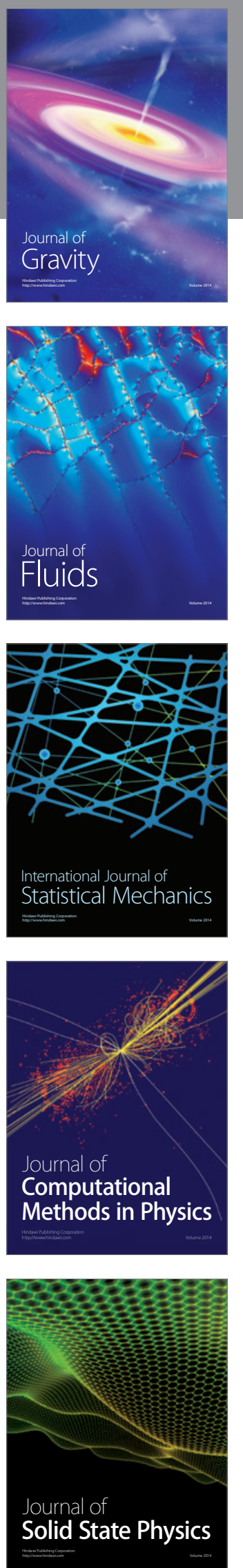

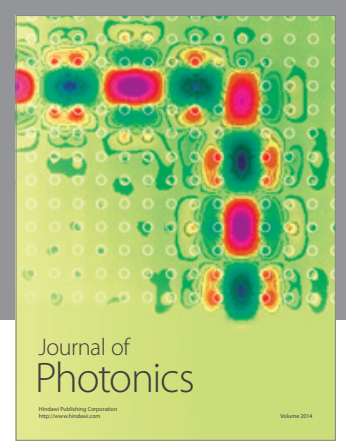

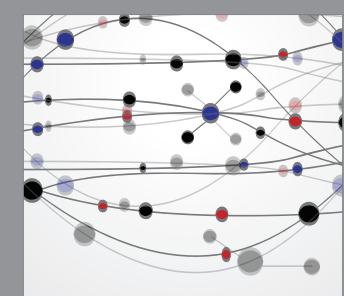

The Scientific World Journal
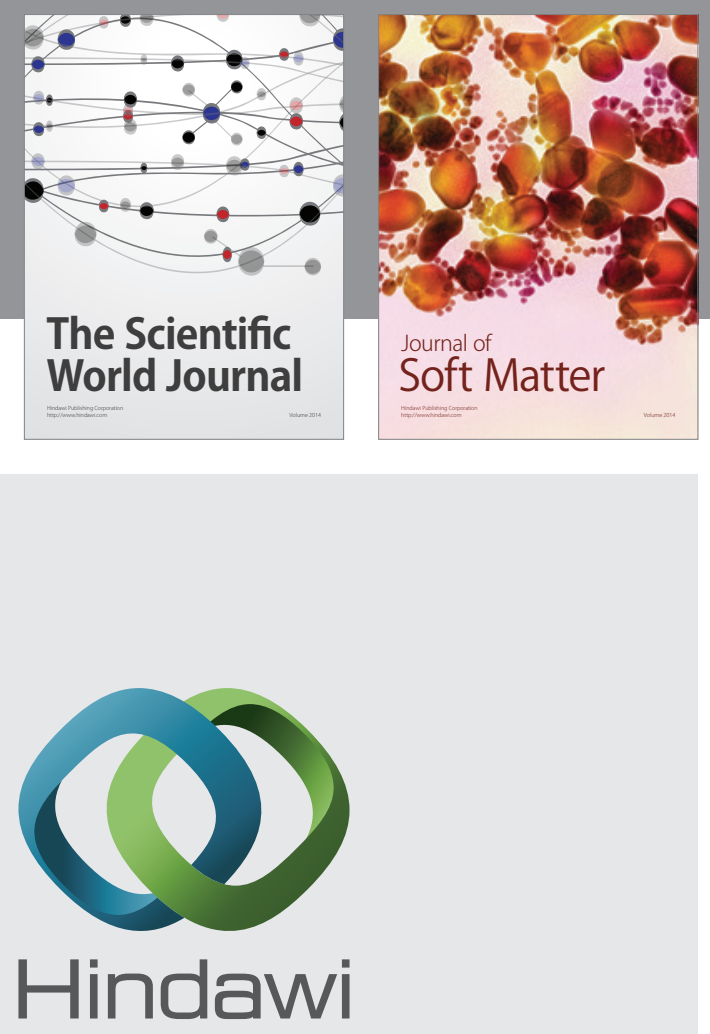

Submit your manuscripts at

http://www.hindawi.com
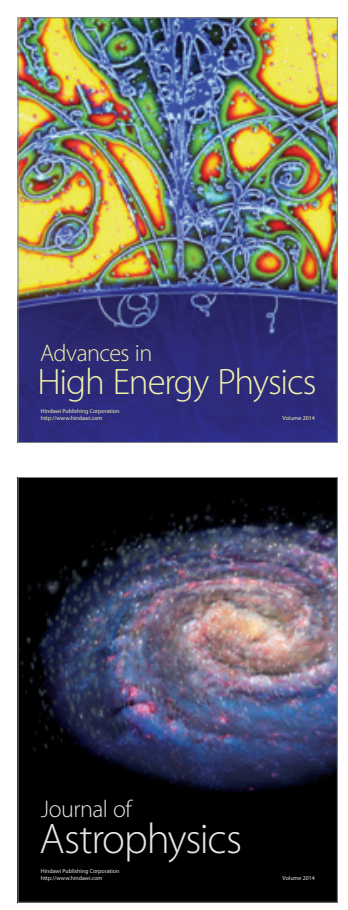
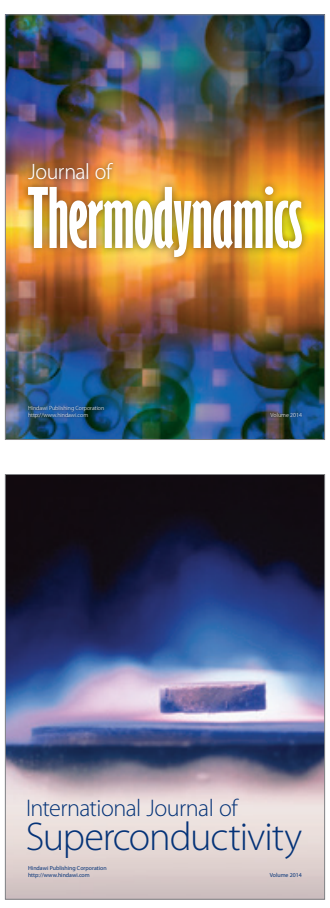
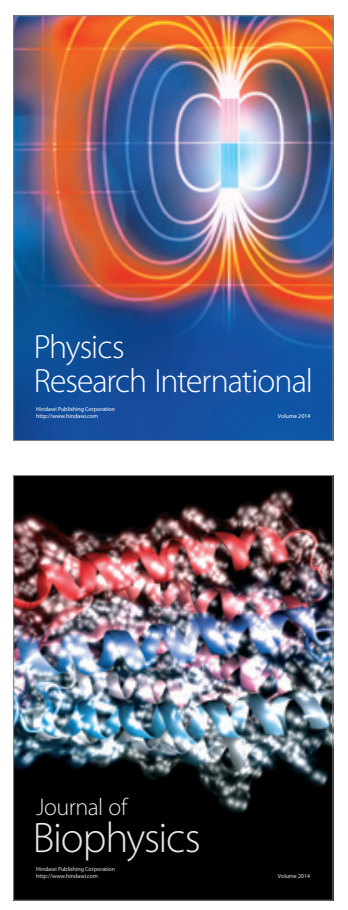
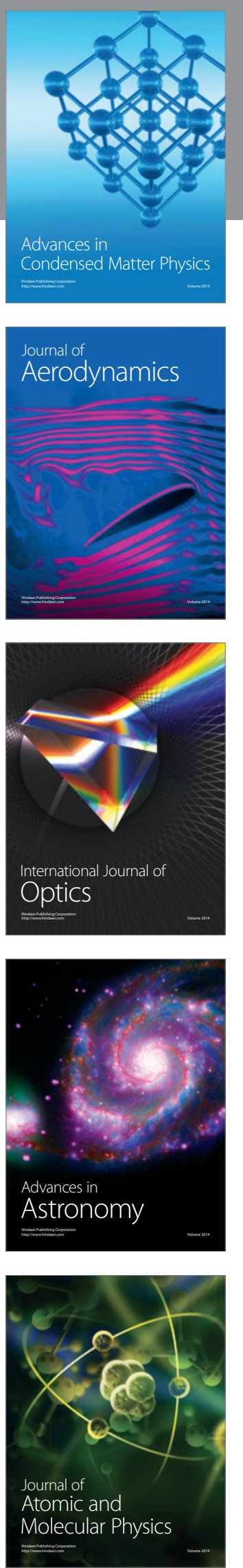